\title{
An experimental investigation for electric discharge grinding chemical vapor deposition silicon carbide
}

\author{
Qidong Geng ${ }^{\mathrm{a}}$, Chunyan $\mathrm{Li}^{\mathrm{b}}$ and Jun Wang ${ }^{\mathrm{c}}$ \\ School of mechanical engineering, Yancheng Institute of Technology, Yancheng 224051, China \\ akengqidong@163.com, bxxmoob@163.com, cwangjun55210@163.com
}

Keywords: electric discharge grinding, copper ring electrode, silicon carbide.

\begin{abstract}
Silicon carbide ( $\mathrm{SiC}$ ) is a kind of material with high specific stiffness, thermal conductivity and low thermal deformation coefficient, which is currently in active demand in aeronautic and astronautic industries. However, the beneficial properties of high hardness and high strength cause problems in shaping SiC blanks. Electrical discharge machining (EDM) is one of the most popular unconventional machining processes used for producing hard and brittle material. But, the processing efficiency of EDM is lower than electrical discharge grinding (EDG). An experimental investigation was made into the improved technologies of EDM. The normal EDM material removal rate (MRR) could be achieved in EDG under suitable conditions of electrode rotating speed, ring copper electrode with a rotating device was made and employed to study the EDG technology.
\end{abstract}

\section{Introduction}

Silicon carbon ( $\mathrm{SiC}$ ), combining high strength and high elastic modulus, is used widely in aeronautic and astronautic industries. Additionally, it has a high melting temperature, low thermal expansion coefficient, and high thermal conductivity. Because of these excellent properties, SiC has been recognized as future materials for reflection mirror. There are numerous types of silicon carbon in the world.

There are many routes of the fabrication of silicon carbon such as hot pressing, reaction bonding, gas phase reaction and chemical vapor deposition. A chemical vapor deposition (CVD) technique is one of the most familiar gas phase reaction methods for the synthesis of highly crystalline, stoichiometric, high-purity ß-SiC. The CVD process can produce a solid SiC form from a gas phase reactant at comparatively low temperature without the use of sintering aids. CVD SiC is known as very difficult-to-machine materials. The main factors that cause CVD SiC to be difficult to machine are its high hardness, high strength, and brittleness. Electrical discharge machining is a non-conventional machining process where material is removed by a succession of electrical discharges occurring between an electrode and a work piece .However, low machining efficiency is one of the main EDM disadvantages. The topic of how to reduce machining time and to maintain reasonable accuracy has always been of research interest. In the recent past, researchers turned to improve the efficiency and quality of electrical discharge machining. Y. Zhao et al developed a foil EDM method to cut a single crystal silicon carbon [1]. Gangaram Mandaloi et al gave a detailed investigation on the crystalline structure of AISI M2 steel by using copper alloy electrode in EDM process [2].

In the EDG process, a copper ring electrode is used and CVD SiC as work piece is reciprocating motion Electrical discharge grinding is a good method of processing CVD SiC.

\section{Principle for EDG of SiC}

The principle of electrical discharge grinding is shown in Fig.1.The tool and the work piece are connected to the positive and negative poles of the pulse generator respectively. Tool electrode is produced by the swivel drive the rotary motion, complete the tool electrode feed servo system, work piece by rotary table driven rotary motion, rotary table is installed in the workbench can be finished around mobile. Tool electrode pulse power at one end, the other end after machining. In EDM 
generating machining, liquid device complete liquid of electrode [1]. Several kinds of discharge in the EDM grinding: open, normal discharge, arc discharge, short circuit. Their features are: open circuit, a single pulse duration did not produce discharge between tool electrode and work piece, machining gap at the ends of the voltage and pulse voltage is consistent, the erosion rate is zero. Normal discharge is an effective state of processing, high frequency wave, often appear on the discharge voltage waveform have breakdown time delay phenomenon, the corrosion rate is generally higher, and won't burn artifact, is the ideal processing status. Arc discharge electrode gap is too small, cannot achieve timely deionization, stable arc, arc stability to maintain voltage and arc transition, almost no breakdown time delay. It will cause the work piece burn, so should avoid as far as possible. Short circuit, small clearances or equal to zero, short circuit, electrode and work piece zero voltage, electric current is the largest. When short circuit happened, it kept not removing steadily.



Fig. 1. Schematic experimental setup for EDG process

\section{Experimental study of EDG of SiC}

In this section, the fundamental EDG behaviors of grinding $\mathrm{SiC}$ were studied. The experiments were conducted on transformational milling machine.The tool electrode was a ring which used copper. It was fixed by milling chuck and servo fed to grinding the work piece. The electrode was fed by a stepper motor. A red copper ring electrode was used on girth to grind the work piece .The radius of the electrode was twenty-five mm. The grade of copper was used of $\mathrm{T} 2$. Commercial kerosene was circulated as the dielectric fluid. The dielectric fluid was injected at a pressure of $3.0 \mathrm{~kg} / \mathrm{m} 2$ through the red copper ring electrode. The work piece was chemical vapor deposition (CVD) SiC with electric resistivity of $0.012 \sim 0.025 \Omega \mathrm{cm}$. The main machining conditions are shown in Table 1 . The machining properties such as material removal rate (MRR), tool wear rate (TWR), surface quality (SQ), surface roughness (SR) and heat affected zone (HAZ). MRR is proportional to the product of the energy transferred per pulse and the pulse frequency. Changing the pulse current at a constant frequency varies the energy of the pulse. Hence all the experiments are conducted with pulse current, pulse duration, volume percentage of $\mathrm{SiC}$, feed speed and speed of the electrode as variables. The pulse

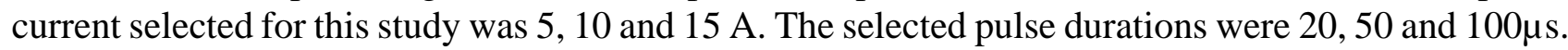
The ring electrodes are rotated at 100,300 and $500 \mathrm{rpm}$. The feed speed of the work piece are 3,6and $9 \mathrm{~m} / \mathrm{s}$. The material removal rate and electrode wear rate were evaluated for each cutting condition by measuring the average amount of the material removed and the required cutting time. Changes in the tool weight, material weight, and elapsed time were recorded after each machining. The MRR and EWR were measured by using an electronic balance of sensitivity $0.1 \mathrm{mg}$. The surface roughness of the CVD SiC was measured with the help of MARSURF M300. The Ra values are used to quantify the surface roughness. The cutoff length for each measurement was taken as $1.0 \mathrm{~mm}$. A mechanism was developed to rotate the tool. The electrode was rotated and sunk simultaneously into the work piece. A stepper motor was used to rotate the work piece (tool). The electrode was controlled with the help of a regulated power supply. A mechanical tachometer was used to measure the speed of the rotating electrode. Experiments are conducted to identify the variables that are likely to have significant influence on the response variable. In this study, MRR, TWR and SR are considered as response variables. These values are compared for stationary, rotating electrode and feeding work piece. Table 1 shows the experiment conditions of EDG. 
Table 1. Experiment conditions

\begin{tabular}{cc}
\hline Work piece & CVD SiC \\
Electrode & Copper ring \\
Open voltage & $100[\mathrm{~V}]$ \\
Preset discharge current & $5,10,15[\mathrm{~A}]$ \\
Discharge duration & $20,50,100[\mu \mathrm{s}]$ \\
Discharge interval & $30,50,100[\mu \mathrm{s}]$ \\
Dielectric fluid & $\mathrm{EDM}$ oil \\
\hline
\end{tabular}

\section{Results and discussion}

Effect of discharge current on EDG. EDG machining performance depends on the gap between electrode and work piece, the discharge current, breakdown voltage, pulse duration, dielectric strength and electrode polarity[Shu and Tu [1]] .For the sake of simplification ,numerous current values were selected and other parameters were fixed for the experiments. Fig. $x$ shows the effect of varying discharge current on material removal rate with a copper ring electrode. The results indicate that the discharge gap width increase as the current increase, and their relationship for $100 \mu$ s pulse duration approaches the following equation.

Gap width $(\mu \mathrm{m})=3.9 \mathrm{~A}+15.0$ where $\mathrm{A}$ is the working current.

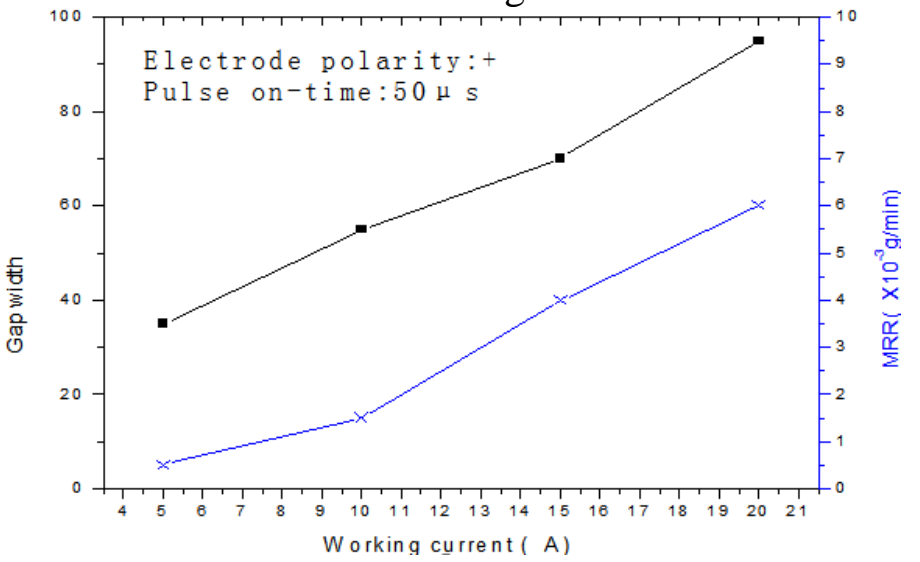

Fig.2. Effect of current on gap width and MRR using copper ring electrode.

Material removal rate is one of the important parameters for evaluating the EDG machining performance for a particular working setup. Fig. 5 also shows the variation of current vs. MRR. The results also indicate that the MRR increases as the current increases and their relationship approaches the following equation

MRR=1.7831e0.0293A

As yet, no expression has been depicted that can predict fully the rate of metal removal in EDM. This rate depends on a large number of properties of the work piece material, including its melting point, thermal conductivity and latent heat. It is also influenced by the properties of the electrodes, and by the geometric factors such as the shape and the dimensions of electrode and work piece.

Effects of electrode rotating speed. The results in Fig. 3 show the effect of electrode rotating speed on surface roughness with ring copper electrodes at 7A working current. It demonstrates that the MRR of EDG, using ring copper electrodes, increase as the rotating speed increases. This is attributed to the fact that when the rotating speed increases, the debris fall off rapidly from the work piece. The gap between work piece and electrode become evenly quickly. The surface roughness, using the EDG machining, is lower than using the EDM machining.

Effects of work piece feeding speed. The results in Fig. 4 show the effect of the work piece on MRR. In the experiment, reciprocating feed method was adopted. It is helpful to improve material removal rate by increasing the feed speed. Electro discharge machining becomes very steadily by rotating electrode and reciprocating feed. Consequently the short circle and arc are avoided by EDG machining. 


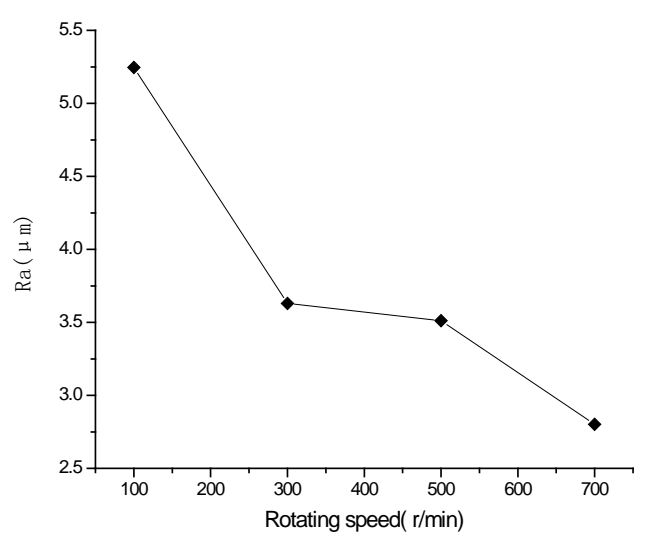

Fig.3.Effect of Rotating speed

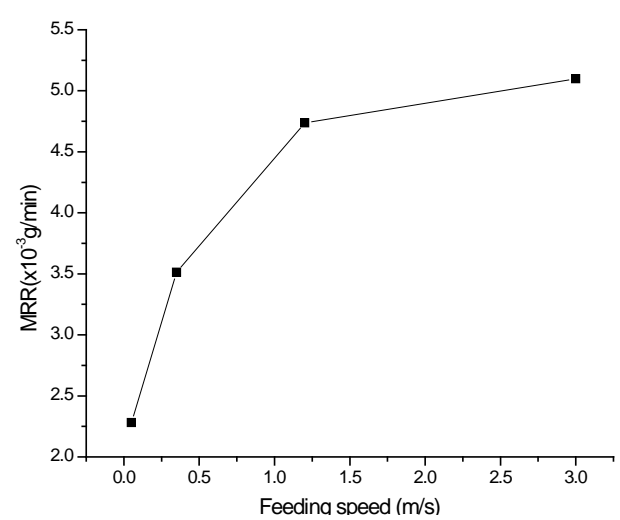

Fig.4.Effect of feeding speed

\section{Conclusions}

An experimental study on EDG has been carried out. From the experiments and research described above, the following conclusions are drawn:

The electrical discharge grinding could be performed by using an electrode rotating facility and copper ring electrodes. The machining is simple in principle, with good MRR, lower surface roughness and low equipment cost.

EDG can obtain high MRR while suitable electrode rotating speed, feeding speed and current are chosen. When the electrode rotating speed is high enough to let hump be ground away before solidification, much higher MRR can be achieved. The MRR can also be improved is near the sum of the gap width and white layer thickness.

Experimental results showed that the EDG machining efficiency is two to three times that of normal EDM operation. In addition, the corresponding surface roughness of work piece after EDG operation is lower than that after EDM operation, this was also caused by the grinding function introduced in EDG operation on work piece surface.

\section{References}

[1] Zhao, Y., M. Kunieda, and K. Abe, Experimental Investigations into EDM Behaviors of Single Crystal Silicon Carbide. Procedia CIRP, 2013. 6: p. 135-139.

[2] Mandaloi, G., et al., Effect on crystalline structure of AISI M2 steel using copper electrode through material removal rate, electrode wear rate and surface finish. Measurement, 2015. 61: p. 305-319.

[3] Shu, K.M. and G.C. Tu, Study of electrical discharge grinding using metal matrix composite electrodes. International Journal of Machine Tools and Manufacture, 2003. 43(8): p. 845-854.

[4] Hsue, A.W.J., J.J. Wang, and C.H. Chang, Milling Tool of Micro-EDM by Ultrasonic Assisted Multi-axial Wire Electrical Discharge Grinding Processes. Proceedings of the Same International Manufacturing Science and Engineering Conference, 2012, 2012: p. 473-479.

[5] Satyarthi, M.K. and P.M. Pandey, Comparison of EDG, Diamond Grinding, and EDM Processing of Conductive Alumina Ceramic Composite. Materials and Manufacturing Processes, 2013. 28(4): p. 369-374. 